Vaccine Volume 24, Issues 44-46, 10 November 2006, Pages 6664-6669

Proceedings of the Second European Influenza Conference, Proceedings of the

Second European Influenza Conference

doi:10.1016/j.vaccine.2006.05.049

Copyright (c) 2006 Elsevier Ltd All rights reserved.

\title{
Use of oseltamivir in Dutch nursing homes during the 2004-2005 influenza season
}

\section{Marianne A.B. van der Sande ${ }^{a}, *$, Wilhelmina L.M. Ruijs ${ }^{b}$, Adam Meijer $^{c, d}$, Herman J.M. Cools ${ }^{e, f}$ and Simone M. van der Plas ${ }^{a}$}

${ }^{a}$ Centre for Infectious Disease Epidemiology, National Institute for Public Health and the Environment (RIVM), Bilthoven, The Netherlands

${ }^{\mathrm{b}}$ National Coordinating Structure Infectious Disease Control, RIVM, Bilthoven, The Netherlands

'Laboratory for Infectious Disease Diagnostics and Screening, RIVM, Bilthoven, The Netherlands

${ }^{\mathrm{d} E u r o p e a n}$ Influenza Surveillance Scheme, Netherlands Institute for Public Health Research, Utrecht, The Netherlands

eDutch Association Nursing Home Clinicians, Utrecht, The Netherlands

fDepartment of General Practice and Nursing Home Medicine, Leiden University

Medical Centre, Leiden, The Netherlands

* Corresponding author. Tel.: +31 30274 3770; fax: +31 302744409.

\begin{abstract}
To assess the implementation of guidelines for using neuraminidase inhibitors in the control of influenza outbreaks in Dutch nursing homes, data were collected on prophylactic and therapeutic use of anti-viral medication, indications for use and criteria for prescribing, based on experiences during the influenza season 20042005 in a retrospective cross-sectional survey among Dutch nursing homes after the 2004-2005 season.
\end{abstract}

Ninety/194 (49\%) participating nursing homes reported an outbreak of influenzalike illness; in 57/194 (29\%) influenza was laboratory confirmed. In 37/57 homes $(65 \%)$ oseltamivir had been used as prophylaxis. Prophylactic use was extended to all residents and staff in $6 / 37(16 \%)$ of homes, but limited in the others. In $9 / 37(24 \%)$ no staff were issued prophylaxis. Among clinicians with laboratory confirmed influenza, 41/46 (89\%) had used oseltamivir therapeutically. Main reasons for not prescribing oseltamivir for prophylaxis and/or therapy were lack of scientific evidence, high costs, and absent or delayed laboratory confirmation.

Logistical bottlenecks in diagnosis, cost-effectiveness concerns, and lack of an evidence-base hamper full integration in policy and should be addressed.

\section{Introduction}

Every year, human influenza virus infections lead to considerable morbidity and mortality among frail and elderly populations [1]. Seasonal variation depends on the virulence of the circulating virus strains and the amount of pre-existing (partial) immunity. Pre-season vaccination is currently the main preventive intervention, both for individual protection and to curb spreading of an outbreak. 
Vaccine Volume 24, Issues 44-46, 10 November 2006, Pages 6664-6669

Proceedings of the Second European Influenza Conference, Proceedings of the Second European Influenza Conference

However, vaccination has only limited effectiveness among the elderly population related to the continuous antigenic drift of the virus in general and a decreased immune response to the vaccine with increasing age and with the occurrence of specific immune-suppressive co-morbidity [2]. Furthermore, immunity wanes over time during in the influenza season.

If despite vaccination an influenza outbreak occurs, antiviral treatment could offer an opportunity to reduce the duration and severity of disease, as well as to limit further spread of an outbreak [3]. The first generation antivirals (amantadine and rimantadine) were active against influenza A only. Widespread use has been further limited due to frequent central nervous system adverse effects and rapid development of resistance. With the development of neuraminidase inhibitors, a new class of antivirals has become available which has fuelled interest in their therapeutic use and also as secondary prevention through post-exposure prophylaxis (PEP). So far, few contra-indications and side effects have been reported, and hardly any drug resistance. Oseltamivir looks particularly promising as it can be taken orally, is licensed for therapeutic and prophylactic use (both in contrast to zanamivir, the other neuraminidase inhibitor available) and is active against influenza A and B (in contrast to the first generation antivirals) [4].

In 2004, the Dutch association of nursing home physicians (NVVA) and the national coordinating structure infectious disease control (LCI) issued guidelines in which they cautiously supported the use of oseltamivir both therapeutically and prophylactic for affected units of care in case of an influenza outbreak in a nursing home [5] and [6]. These guidelines led to much discussion in the Dutch medical community. This paper reports on the evaluation of the implementation of the guidelines in Dutch nursing homes during the 2004-2005 influenza season, and their role in the control of influenza outbreaks.

\section{Materials and methods}

In 2004-2005, the influenza season in the Netherlands occured between week 44, 2004 and week 12, 2005. After a pilot phase, all 348 Dutch nursing homes were sent a questionnaire in April 2005, at the end of the season.

Data were collected on characteristics of the nursing home (size, vaccination coverage) and occurrence of one or more (clinical) influenza outbreaks in the 2005-2005 season. If an outbreak had occurred, further details were asked, including the use of antiviral medication. If no or only partial antiviral medication was used during an outbreak, reasons for not using them were assessed, both for therapeutic and prophylactic use. Furthermore, clinicians were asked to score criteria for prescription on a scale between 1 ('of no importance') and 5 ('of great importance').

A telephonic follow-up was conducted among a $25 \%$ sample of final nonresponders.

\subsection{Definitions}

A confirmed outbreak was defined as the occurrence of more than one influenzalike illness case in a unit of care, of which at least one was laboratory confirmed.

A unit of care was defined as a set group of residents who are under the care of a fixed group of staff (both permanent and temporary). 
Vaccine Volume 24, Issues 44-46, 10 November 2006, Pages 6664-6669 Proceedings of the Second European Influenza Conference, Proceedings of the Second European Influenza Conference

Data were entered in Microsoft Access 2002, and analysed using Stata 8.0 (Stata Corp, TX, USA). Proportions were compared using chi-square testing, differences between medians were assessed using Wilcoxon testing.

Ethical approval was not indicated as this study involved retrospective data collection at institution level only.

\section{Results}

\subsection{Nursing homes}

Information for $194 / 348$ (56\%) of nursing homes was received by questionnaire. In 95/194 (49\%) homes, a clinical outbreak was observed in the 2004-2005 season. Table 1 summarizes baseline characteristics of the participating homes. There was no difference in vaccination coverage of residents or staff between houses with and without an outbreak.

Table 1.

Baseline characteristics of participating nursing homes, by reported influenza outbreak in the 2004-2005 season (median, inter quartile range)

\begin{tabular}{|l|l|l|l|}
\hline Category & Clinical outbreak & No outbreak & $\boldsymbol{p}$ \\
\hline$n$ & 95 & 99 & \\
\hline Total number of residents & $184(130-280)$ & $175(120-240)$ & 0.5 \\
\hline Total number of staff & $305(200-460)$ & $262(180-400)$ & 0.4 \\
\hline Vaccination coverage residents (\%) & $90(90-95)$ & $90(88-95)$ & 0.4 \\
\hline Vaccination coverage staff $(\%)$ & $10(5-15)$ & $10(5-20)$ & 0.9 \\
\hline Number of units of care & $7(5-11)$ & $7(5-11)$ & 1.0 \\
\hline Number of clinicians & $4(3-7)$ & $4(3-5)$ & 0.8 \\
\hline Years of experience as clinician & $16(12-23)$ & $16(13-21)$ & 0.9 \\
\hline
\end{tabular}

\subsection{Influenza outbreaks}

The median number of units involved was 2 (range 1-12). The median number of affected residents was 14 (range 1-84), and of affected staff 12 (range 2-68).

In $64 / 95(67 \%)$ nursing homes with a reported clinical outbreak, laboratory tests were requested for virological confirmation. In none of the nursing homes a nearpatient test was used to seek virological confirmation. The majority (54/64, 84\%) had requested a 'fast track' laboratory test (which could be a variety of tests including PCR) from a nearby laboratory, sometimes combined with other more time-consuming diagnostics, such as culture. In 7/64 (11\%) homes, the diagnosis of influenza was not virologically confirmed. Thus, overall 57/194 (29\%) of Dutch 
Vaccine Volume 24, Issues 44-46, 10 November 2006, Pages 6664-6669 Proceedings of the Second European Influenza Conference, Proceedings of the Second European Influenza Conference

nursing homes had a laboratory confirmed influenza outbreak during the 20042005 season.

\subsection{Anti-viral prophylaxis}

In $37 / 57$ (65\%) nursing homes with a laboratory confirmed outbreak, antiviral PEP was prescribed, as well as in one nursing home in the absence of virological confirmation. The median duration of PEP was 8 days for residents (range 2-14) and 9 days for staff (range 7-14). Start of PEP for residents occurred after a median of $48 \mathrm{~h}$ (range 4-336), and for staff after a median of $66 \mathrm{~h}$ (range 16336) following the start of the outbreak. All but one of the homes used oseltamivir, the exception used amantadine (Table 2).

Table 2.

Reasons for not prescribing antiviral post-exposition prophylaxis during an influenza outbreak $(n, \%)$

\begin{tabular}{|l|l|l|l|l|}
\hline & Total & $\begin{array}{l}\text { Confirmed } \\
\text { outbreak }\end{array}$ & $\begin{array}{l}\text { No } \\
\text { (confirmed) } \\
\text { outbreak }\end{array}$ & $\boldsymbol{p}$ \\
\hline Nursing homes $^{\mathrm{a}}$ & 136 & 57 & 79 & \\
\hline No influenza on unit & $86(63)$ & $27(47)$ & $59(75)$ & 0.001 \\
\hline Lack of evidence-base & $46(34)$ & $16(28)$ & $30(38)$ & 0.2 \\
\hline Influenza mild & $43(32)$ & $14(25)$ & $29(37)$ & 0.1 \\
\hline Costs & $42(31)$ & $16(28)$ & $26(33)$ & 0.7 \\
\hline No or late laboratory confirmation & $41(30)$ & $11(19)$ & $30(38)$ & 0.02 \\
\hline No operational outbreakplan & $36(26)$ & $9(16)$ & $27(34)$ & 0.02 \\
\hline Other prevention & $30(22)$ & $26(46)$ & $4(5)$ & $<0.001$ \\
\hline Should be implemented by GP & $24(18)$ & $10(18)$ & $14(18)$ & 1.0 \\
\hline Vaccination should be sufficient & $22(16)$ & $5(9)$ & $17(22)$ & 0.05 \\
\hline Side-effects & $17(13)$ & $11(19)$ & $6(8)$ & 0.04 \\
\hline Ethical objections & $16(12)$ & $5(9)$ & $11(14)$ & 0.4 \\
\hline Lack of support among staff ${ }^{b}$ & $16(12)$ & $10(18)$ & $6(8)$ & 0.08 \\
\hline Logistical impediments & $16(12)$ & $8(14)$ & $8(10)$ & 0.5 \\
\hline Other reasons & $21(16)$ & $17(30)$ & $4(5)$ & $<0.001$ \\
\hline
\end{tabular}

${ }^{a}$ More than one reason per nursing home possible.

${ }^{b}$ Relates to prophylaxis of staff only. 
Vaccine Volume 24, Issues 44-46, 10 November 2006, Pages 6664-6669 Proceedings of the Second European Influenza Conference, Proceedings of the Second European Influenza Conference

In $9 / 37$ (24\%) homes, PEP prescription was not extended to staff and limited to residents only. PEP was usually limited to the affected units $(28 / 37,76 \%)$, although in 6 homes $(16 \%)$ it was offered to all residents and staff of the nursing home. In the remaining homes PEP was offered to some but not all in affected units, related to logistical and medical reasons. The median number of residents receiving PEP was 50 (range 4-150), and of staff 40 (range 10-292). Reasons for not prescribing PEP to all or some residents and staff are summarised in Table 3, as reported by homes with and without a confirmed outbreak. Clinical judgement on the severity of the outbreak was a strong factor, while lack of evidence on efficacy in nursing homes, high costs, and uncertainty of the diagnosis in the absence of rapid laboratory confirmation, were important reasons not to prescribe antiviral PEP. The importance given to the presence of an operational outbreak plan to address the occurrence of an outbreak supports the need for early planning and preparation. Both gastro-intestinal side-effects and allergic skin reactions were mentioned as potential adverse effects.

Table 3.

Reasons for not prescribing antiviral therapy during an influenza outbreak $(n, \%)$

\begin{tabular}{|l|l|l|l|l|}
\hline & Total & $\begin{array}{l}\text { Confirmed } \\
\text { outbreak }\end{array}$ & $\begin{array}{l}\text { No } \\
\text { (confirmed) } \\
\text { outbreak }\end{array}$ & $\boldsymbol{p}$ \\
\hline Nursing homes $^{\mathrm{a}}$ & 49 & 23 & 26 & \\
\hline Clinical judgement & $27(55)$ & $16(70)$ & $11(42)$ & 0.06 \\
\hline No operational outbreakplan & $17(35)$ & $2(9)$ & $15(58)$ & $<0.001$ \\
\hline No or late laboratory confirmation & $15(31)$ & $1(4)$ & $14(54)$ & $<0.001$ \\
\hline Ethical objections & $10(20)$ & $6(26)$ & $4(15)$ & 0.4 \\
\hline Costs & $3(6)$ & $2(9)$ & $1(4)$ & 0.5 \\
\hline Side-effects & $2(4)$ & $2(9)$ & 0 & 0.1 \\
\hline Other reasons & $6(12)$ & $5(22)$ & $1(4)$ & 0.06 \\
\hline
\end{tabular}

${ }^{a}$ More than one reason per nursing home possible.

It should be noted that 56/57 (98\%) of nursing homes with, and 39/137 (29\%) of nursing homes without a confirmed outbreak implemented other preventive measures to reduce the impact of an outbreak. These preventive measures included increasing awareness among staff $(62 \%)$, introduction of cohort nursing $(57 \%)$, physical separation of residents $(38 \%)$ and cancellation of group activities $(53 \%)$, wearing of facial masks (53\%), and intensified manual hygiene $(66 \%)$.

\subsection{Antiviral therapy}

Seventy-four/200 (37\%) participating clinicians had diagnosed influenza on their units of care, and $52 / 74$ (70\%) had asked for laboratory confirmation. Fortysix/52 (88\%) clinicians had their clinical diagnosis of influenza virologically confirmed. Most of these clinicians $(41 / 46,89 \%)$ with confirmed influenza had 
Vaccine Volume 24, Issues 44-46, 10 November 2006, Pages 6664-6669

Proceedings of the Second European Influenza Conference, Proceedings of the Second European Influenza Conference

prescribed antiviral therapy, as well as six clinicians who did not have laboratory confirmation of the outbreak. All clinicians prescribed oseltamivir. Median duration of therapeutic use was 5 days (range 2-11), which was started after a median of $24 \mathrm{~h}$ following the clinical diagnosis.

Half $(23 / 46,50 \%)$ of the clinicians with a confirmed outbreak on one of their units, prescribed antiviral therapy to all their patients on an affected unit, 5 $(11 \%)$ to none of their patients, and the others $(18,39 \%)$ to some patients. The main reasons for not prescribing antiviral mediation to (some) patients are summarised in Table 4. Included are answers from five clinicians who prescribed some oseltamivir despite a negative laboratory diagnosis, as well as from 21 clinicians who suspected influenza but did not ask for laboratory confirmation. Clinicians whose clinical diagnosis was not supported by a virological diagnosis indicated that the lack of laboratory confirmation and the lack of an operational outbreak plan were major factors for not prescribing antiviral therapy, while for those with laboratory confirmation, ethical reasons were a major factor. 
Vaccine Volume 24, Issues 44-46, 10 November 2006, Pages 6664-6669

Proceedings of the Second European Influenza Conference, Proceedings of the

Second European Influenza Conference 
Vaccine Volume 24, Issues 44-46, 10 November 2006, Pages 6664-6669

Proceedings of the Second European Influenza Conference, Proceedings of the Second European Influenza Conference

Table 4.

Importance allocated to specified criteria which influence the decision in Dutch nursing homes to use antivirals as prophylaxis or therapy, ordered by median weight (scale $1-5)$

\begin{tabular}{|c|c|c|}
\hline & Prophylaxis & Therapy \\
\hline \multicolumn{3}{|l|}{ Important or very important (score $\geq 4$ ) } \\
\hline Presence of more than one clinical influenza patient, with at least one virological confirmation within the unit of care within $48 \mathrm{~h}$. & 5 & 5 \\
\hline Number of laboratory confirmed influenza patients within the nursing home & 5 & 4 \\
\hline Number of laboratory confirmed influenza patients within the unit of care & 5 & 4 \\
\hline Number of clinical influenza patients (residents and staff) within the unit of care & 5 & 3 \\
\hline Presence of an influenza outbreak plan in the nursing home & 4 & 4 \\
\hline Presence of more than one clinical influenza patient, with at least one virological confirmation within the nursing home within $48 \mathrm{~h}$ & 4 & 4 \\
\hline Laboratory confirmation of the patient & - & 5 \\
\hline Severity of clinical influenza of the patient & - & 4 \\
\hline Opinion of the patient and/or family & - & 4 \\
\hline \multicolumn{3}{|l|}{ Some importance (score $>2$ but score $<4$ ) } \\
\hline Number of clinical influenza patients (residents and staff) within the nursing home & 4 & 3 \\
\hline Number of staff on sick leave within the unit of care, possible related to influenza & 4 & 3 \\
\hline Experiences during previous influenza outbreaks & 4 & 3 \\
\hline
\end{tabular}


Vaccine Volume 24, Issues 44-46, 10 November 2006, Pages 6664-6669

Proceedings of the Second European Influenza Conference, Proceedings of the Second European Influenza Conference

\begin{tabular}{|c|c|c|}
\hline & Prophylaxis & Therapy \\
\hline Accuracy of match between the vaccine and circulating virus & 4 & 3 \\
\hline Awareness of the severity of the epidemic in the outside community & 4 & 3 \\
\hline Baseline clinical profile (including age) of patients on the unit of care & 3 & 4 \\
\hline Number of staff on sick leave within the nursing home, possible related to influenza & 3 & 3 \\
\hline Known influenza outbreaks in other nursing homes in the same season & 3 & 3 \\
\hline Residents use common spaces & 3 & - \\
\hline Ongoing communal activities of residents & 3 & - \\
\hline Other preventive measures in nursing home or unit & 3 & - \\
\hline Absence of cohort nursing & 3 & - \\
\hline Increased mortality on unit of care & - & 3 \\
\hline Vaccination coverage among residents on unit of care & 3 & 2 \\
\hline Vaccination coverage among staff on unit of care & 3 & 2 \\
\hline Costs for nursing home & 3 & 1 \\
\hline \multicolumn{3}{|l|}{ Little of no importance (score $\leq 2$ ) } \\
\hline Type of unit of care & 2 & 2 \\
\hline Absence of facial masks being worn by staff & 2 & - \\
\hline Vaccination status of patient & - & 2 \\
\hline
\end{tabular}




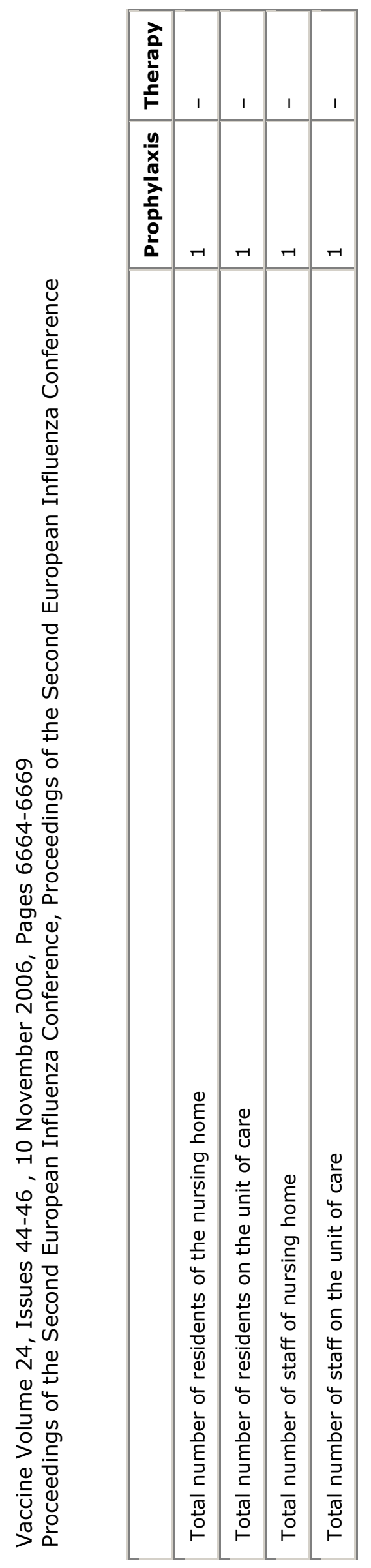


Vaccine Volume 24, Issues 44-46, 10 November 2006, Pages 6664-6669

Proceedings of the Second European Influenza Conference, Proceedings of the Second European Influenza Conference 
Vaccine Volume 24, Issues 44-46, 10 November 2006, Pages 6664-6669

Proceedings of the Second European Influenza Conference, Proceedings of the Second European Influenza Conference

\subsection{Criteria for prescribing of antivirals}

Table 4 gives an overview of the weight given to a set of specified criteria which could impact on the decision to implement the use of antivirals. Most, but not all, criteria could be scored for both PEP and therapeutic use. For prophylactic use, most weight was given to clinical and laboratory confirmation of influenza on the unit of care. For therapeutic use, laboratory confirmation of the patient was given most weight. Apart from the weight given to costs, there was little difference between the weight of criteria in using antivirals for PEP or for therapy.

\subsection{Qualitative feedback}

Several nursing homes gave additional comments on the use of antivirals, which could be divided into categories:

(a) Official guidelines: 'lack of consultation before implementation', perceived 'inconsistencies', 'vagueness' or 'gaps' in the guideline, 'difficult to implement'.

(b) Outbreak plan: 'in preparation', 'modified' from official guidelines.

(c) Ethical objections: 'medicalisation', 'moral pressure on staff'.

(d) Priorities in care: 'biscuits versus oseltamivir', 'increased workload versus limited reduction in disease duration'.

\subsection{Non-responders}

Forty/153 (25\%) non-responders were contacted by telephone as follow-up. The main reason for non-response given was lack of time (16/40). From six nursing homes, a completed questionnaire was still received and included. Of the nonresponders, $8 / 30(27 \%)$ indicated to have experienced influenza during the 2004-2005 influenza season in their nursing home, which was confirmed for four of them. Three/four nursing homes had used oseltamivir as therapy, and one also as PEP. Reasons mentioned for not prescribing oseltamivir were lack of evidence base $(3 \times)$, costs, lack of an operational outbreak plan, side-effects, and (among staff) lack of support (all $1 \times$ ).

\section{Discussion}

The principal finding of our study was that in spite of significant reservations being raised by over a third of participating nursing homes about the guidelines on antiviral use in nursing homes, nearly $90 \%$ of affected units of care implemented at least some therapeutic use of antivirals and nearly two-thirds of affected homes prescribed at least some PEP with antivirals.

The main concern was the current lack of evidence for the recommendation to prescribe oseltamivir in nursing homes. Studies so far have suggested, but not proven that anti-virals could be effective in nursing homes in reducing transmission when used as PEP [7]. Experimental research [8], observational studies in healthy adults [9] and trials among health family members [10] and [11] did show an effect of oseltamivir in interrupting transmission. A recent observational study in eight nursing homes showed a reduced incidence of influenza among residents who received PEP with oseltamivir [12]. This was not a randomised study however, and reduction of the influenza activity can have 
Vaccine Volume 24, Issues 44-46, 10 November 2006, Pages 6664-6669 Proceedings of the Second European Influenza Conference, Proceedings of the Second European Influenza Conference

coincided with a reduction of the epidemic activity. So far, one randomised trial has been published among elderly subjects who were living in residential housing for senior citizens. In this trial a non-standard duration of PEP (6 weeks) was used and PEP could be initiated following detection of influenza in the vicinity of a home, making these data hard to extrapolate. The current lack of scientific evidence for effectiveness among high-risk groups such as nursing home residents is confirmed in a recent meta-analysis [13].

As observed before, timely implementation of antiviral medication can be a challenge [14]. Only half of the Dutch nursing homes managed to start oseltamivir within $48 \mathrm{~h}$ of the start of symptoms. Improved procedures, which would facilitate rapid laboratory diagnosis, will be instrumental in reducing this time span. Concerns on the need of many of the scarce financial resources has also been vouched in the UK [15], although a recent Canadian analysis observed that use of oseltamivir in nursing homes could be cost-effective [16].

Despite of the high vaccination coverage among residents of around $90 \%$, influenza outbreaks occurred in a large number of nursing homes. Due to the continuous antigenic drift of the influenza virus, some mismatch between the vaccine and the circulating strains will usually occur. Unless the mismatch is considerable as occurred in the 2003/2004 season when the Fujian-like A(H3N2) virus circulated [17] and [18], a limited mismatch is unlikely to result in reduced vaccine efficacy. Among the elderly population, the immune response to vaccination is limited due to age-related decline in immune function, often compounded by specific immune-suppressive pathology. This can result in an average vaccine effectiveness among the elderly population of less than $50 \%$ [19]. If outbreaks occur late in the season, waning immunity further reduces the number op protected people. Also, vaccination coverage among staff was very low, and thus the overall vaccination coverage in many nursing homes may have only been around $50 \%$.

The response rate to the questionnaire was $56 \%$. When including the basic data obtained from the non-responders, $65 \%$ of Dutch nursing homes have been reached. It cannot be excluded that some selection bias has occurred. It is likely that among the non-responders fewer outbreaks have occurred, which may have led to an overestimation of the percentage of nursing homes suffering an outbreak. On the other hand, a third of the nursing homes that suspected an outbreak on clinical grounds, did not request laboratory testing to confirm this clinical diagnosis, which may have led to underestimation of the percentage of confirmed outbreaks. There is no reason to assume the non-responders would represent different opinions regarding the use of antivirals; which is supported by the fact that non-responders interviewed gave similar reasons as those who returned the questionnaire.

In conclusion, in view of the high risk among the frail nursing homes populations and the threat of a possible influenza pandemic, nursing homes should be encouraged to formulate an outbreak management plan well ahead of the expected season. At the same time, this study indicates that the major concerns with regards to the prescription of antivirals in nursing homes need to be addressed if antiviral medication is to play a central role in the control of influenza outbreaks in nursing homes. 
Vaccine Volume 24, Issues 44-46, 10 November 2006, Pages 6664-6669

Proceedings of the Second European Influenza Conference, Proceedings of the Second European Influenza Conference

\section{Acknowledgements}

We are grateful to Marina de Jong (NVVA), Beer Liemeyer (NVVA), Henny van Beusichem (Arcares), Kittie van Schie (Adresboek VVZS) and Inf@ct for support in contacting nursing homes. We like to acknowledge Gabriella Morroy, Yvonne van Duynhoven, Rianne van Gageldonk, Marion Koopmans and Marina Conyn for contributing to the design of the study, and Frederika Dijkstra for critically reviewing an earlier draft of the manuscript. Most of all we are indebted to all the nursing home clinicians who gave some of their precious time to answer our detailed questionnaire.

The study was funded by the Dutch Ministry of Health. The sponsor source had no involvement in study design, methods, data collection, analysis, and drafting of the manuscript.

Contributors: Marianne van der Sande, contributed to design, was responsible for implementation and analysis, and drafted the manuscript. Wilhelmina (Helma) Ruijs, Herman Cools, Adam Meijer, Simone van der Plas contributed to design and implementation of the study, and critical revision of the manuscript. All authors approved the final version of the manuscript.

\section{References}

[1] P.A. Patriarca, J.A. Weber, R.A. Parker, W.N. Hall, A.P. Kendal and D.J. Bregman et al., Efficacy of influenza vaccine in nursing homes. Reduction in illness and complications during an influenza $A$ (H3N2) epidemic, JAMA 253 (1985), pp. 1136-1139.

[2] C.B. Bridges, S.A. Harper, K. Fukuda, T.M. Uyeki, N.J. Cox and J.A. Singleton, Prevention and control of influenza. Recommendations of the Advisory Committee on Immunization Practices (ACIP), MMWR 52 (2003), pp. 1-34.

[3] L.V. Gubareva, L. Kaiser and F.G. Hayden, Influenza virus neuraminidase inhibitors, Lancet 355 (2000), pp. 827-835.

[4] P.H. Peters Jr., S. Gravenstein, P. Norwood, V. De Bock, A. Van Couter and M. Gibbens et al., Long-term use of oseltamivir for the prophylaxis of influenza in a vaccinated frail older population, J Am Geriatr Soc 49 (2001), pp. 1025-1031.

[5] Cools HJM, Herngreen JJ, Jong RE de, Lichtenbelt MF, Rothbarth PH, Essen GA van. NVVA Richtlijn Influenza preventie in verpleeghuizen en verzorgingshuizen; 2004.

[6] Landelijke Coördinatiestructuur Infectieziektebestrijding. LCI protocol influenza (j10-j11) Bijlage IV: Influenza in verpleeg- en verzorgingshuizen, Utrecht; 2004.

[7] S.K. Bowles, W. Lee, A.E. Simor, M. Vearncombe, M. Loeb and S. Tamblyn et al., Use of oseltamivir during influenza outbreaks in Ontario nursing homes, J Am Geriatr Soc 50 (2002), pp. 608-616.

[8] F.G. Hayden, J.J. Treanor, R.S. Fritz, M. Lobo, R.F. Betts and M. Miller et al., Use of the oral neuraminidase inhibitor oseltamivir in experimental human influenza: randomized controlled trials for prevention and treatment, JAMA 282 (1999), pp. 1240-1246.

[9] F.G. Hayden, R.L. Atmar, M. Schilling, C. Johnson, D. Poretz and D. Paar et al., Use of the selective oral neuraminidase inhibitor oseltamivir to prevent influenza, N Eng J Med 341 (1999), pp. $1336-1337$. 
Vaccine Volume 24, Issues 44-46, 10 November 2006, Pages 6664-6669 Proceedings of the Second European Influenza Conference, Proceedings of the Second European Influenza Conference

[10] F.G. Hayden, R. Belshe, C. Villanueva, R. Lanno, C. Hughes and I. Small et al., Management of influenza in households: a prospective, randomized comparison of oseltamivir treatment with or without postexposure prophylaxis, J Infect Dis 189 (2004), pp. 440-449.

[11] R. Welliver, A.S. Monto, O. Carewicz, E. Schatteman, M. Hassman and J. Hedrick et al., Effectiveness of oseltamivir in preventing influenza in household contacts: a randomized controlled trial, JAMA 285 (2001), pp. 748-754.

[12] A.S. Monto, J. Rotthoff, E. Teich, M.L. Herlocher, R. Truscon and H.-L. Yen et al., Detection and control of influenza outbreaks in well-vaccinated nursing home populations, Clin Infect Dis 39 (2004), pp. 459-464.

[13] N.J. Cooper, A.J. Sutton, K.R. Abrams, A. Wailoo, D.A. Turner and K.G. Nicholson, Effectiveness of neuraminidase inhibitors in treatment and prevention of influenza A and B: systematic review and meta-analysis of randomised controlled trials, BMJ 326 (2003), pp. 1235-1240.

[14] P.J. Drinka, P. Krause, L. Nest, S. Gravenstein, B. Goodman and P. Shult, Delays in the application of outbreak control prophylaxis for influenza $A$ in a nursing home, Infect Control Hosp Epidemiol 23 (2002), pp. 600-603.

[15] R. Harling, A. Hayward and J.M. Watson, Implications of the incidence of influenza-like illness in nursing homes for influenza chemoprophylaxis: descriptive study, BMJ 329 (2004), pp. 663-664.

[16] N.A. Risebrough, S.K. Bowles, A.E. Simor, A. McGeer and P.I. Oh, Economic evaluation of oseltamivir phosphate for postexposure prophylaxis of influenza in long-term care facilities, J Am Geriatr Soc 53 (2005), pp. 444-451.

[17] W.J. Paget, T.J. Meerhoff and H. Rebelo de Andrade, Heterogeneous influenza activity across Europe during the winter of 2002-2003, Euro Surveill 8 (2003), pp. 230-239.

[18] Centers for Disease Control and Prevention (CDC). Preliminary assessment of the effectiveness of the 2003-04 inactivated influenza vaccine-Colorado, December 2003. Morb Mortal Weekly Report 2004;53:8-11.

[19] T.M. Govaert, M.J. Sprenger, G.J. Dinant, K. Aretz, N. Masurel and J.A. Knotnerus, Immune response to influenza vaccination of elderly people. A randomized double-blind placebo-controlled trial, Vaccine 12 (1994), pp. 1185-1189. 